
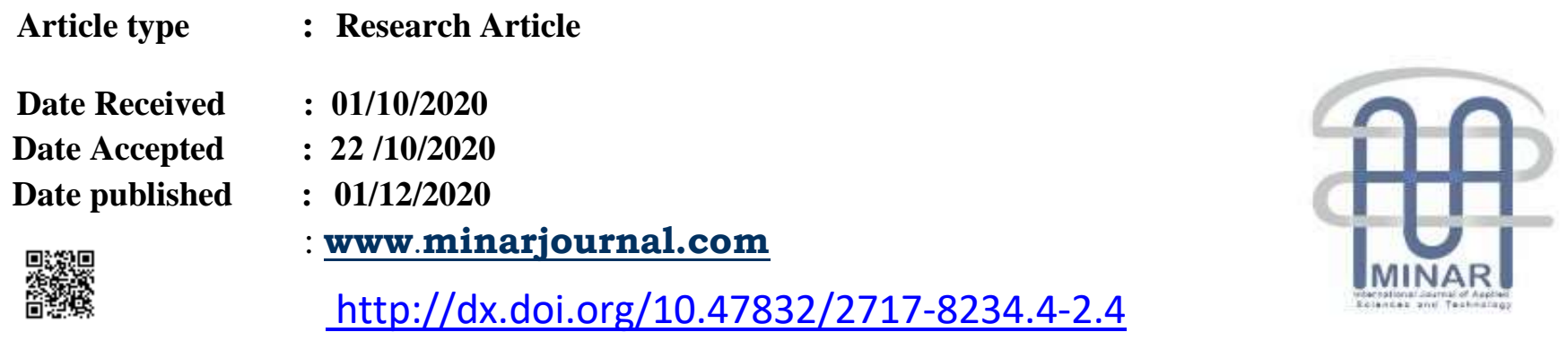

\title{
ELECTRON DENSITY SPECTROSCOPIC MEASUREMENT IN AL LASER INDUCED PLASMA
}

\section{Mohammed R. ABDULAMEER1 ${ }^{1}$, Ali A-K. HUSSAIN ${ }^{2}$, Kadhim A. AADIM3}

\begin{abstract}
Plasma generated by a $1064 \mathrm{~nm}$ pulsed Nd: YAG laser with pulse duration of $10 \mathrm{~ns}$ concentrated onto an Al solid target under vacuum pressure was examined spectroscopically. The temperature and electron density specifying the plasma were measured by time-resolved spectroscopy of neutral atom and ion line emissions in the time period range of 300-2000 ns. An echelle spectrograph is utilized to appear the plasma emission lines. The temperature was obtained using the spectral line comparison method and the electron density was calculated using the Stark Broadening (SB) method. The electron density was characterized as a function of laser pulse energy. The time range where the plasma is optically thin and is also in local thermodynamic equilibrium (LTE), significant for the laser-produced plasma (LPP) which was evaluated from the temporal profile of the intensity ratio of two Al I lines $(\lambda 1=380.581 \mathrm{~nm}$, $398.014 \mathrm{~nm}$, and $393.1996 \mathrm{~nm}),(\lambda 2=586.781 \mathrm{~nm})$. It is found to be $700-1000 \mathrm{~ns}$.

Keywords: Laser Induced Plasma, Spectrometer, Electron Density, Plasma Physics, Plasma Plume.
\end{abstract}

\footnotetext{
${ }^{1}$ Baghdad University, Iraq, mohammed plasma@sc.uobaghdad.edu.iq, https://orcid.org/0000-0002-7395-9840

${ }^{2}$ Baghdad University, Iraq, ali.a@sc.uobaghdad.edu.iq, https://orcid.org/0000-0002-2548-5046

${ }^{3}$ Baghdad University, Iraq, kadhim adem@scbaghdad.edu.iq
} 


\section{Introduction}

In the operation of pulsed laser deposition of solid target، energetic species are emitted from the surface of the target. The energy reaches up to several hundreds of $\mathrm{eV}$. In the plume, the removed particles undergo different chemical reactions by themselves as well as background species [1]. Laser matter interaction has recognized many application on the basis of laser deposition in fundamental studies and technological applications such as thin film deposition, environmental monitoring, biomedical studies,generation of micro clusters, cutting, drilling, surface treatment, laser patterning, manufacturing of micro and nanoelectronic devices and magneto hydrodynamic generators, etc.[2-4]. Laser induced plasma (LIP) technique is relied on optical diagnostic of particular atoms and molecules by observing their emission signals from the laser produced plasma. This technique is very easy as compared to many other types of elemental analysis methods owing to its straightforward experimental set-up [5].Laser solid interaction goes to the generation of laser induced plasma after a number of energy conversions, leading to the energy of incident laser exceeds the ablation threshold of the solid. Plasma ejectants are composed of a combination of atoms, molecules, electrons, ions, clusters, micron-sized particles, and molten globules. The laser energy converted to target material via electrons likes various mechanisms, viz. electron-phonon coupling, multi-photon ionization and consecutive ionization, etc. [2]. In laser produced plasma method, high intense laser pulse interacts with a matter of target resulting in creation of a micro-plasma in nanosecond because of high intensity plume expansion propagate. The primary part of the plasma is re-heated by the inverse bremsstrahlung (IB) absorption mechanism [3-5]. Plasma parameters can be represented by behavior of electrons, i.e. electron density, temperature, ionization degree, ratio of number densities of neutral metal atoms and ions [2]. The nature of the laser-induced plasma from a solid material rely on wavelength, intensity, pulse duration, pulse spot size, energy absorption and transfer, duration of exposure, beside of the chemical composition target material and atmospheric environment such as pressure, space and time [3-5] The minimum fluence needed to detect charged particles in the vaporized material is recognized as the ablation limit, approximately between 1 and $102 \mathrm{~J} / \mathrm{cm} 2$. Successively the vaporized material propagates in vacuum at supersonic speeds. Expansion mechanism of the plasma vaporized cloud in vacuum can be evaluated by analytical models that are qualitatively matched with the experimental results. By increasing the fluence over the ablation threshold, the saturation of the induced plasma density allows the increase of the ions and electrons temperatures and density [6].The laser induced plasma process can be divided into three states: evaporation of the target material, interaction of the evaporated material species with incident laser beam resulting in material cloud heating and plasma formation, and expansion and fast cooling of the plasma. The dynamic, character of the laser induced plasma is challenging for diagnostics because the initial plasma variables differ in time and space $[7,8]$.Analysis of laser induced plasma can be a very confrontation task considering its transient nature beside large variations in plasma properties with space and time. There are different diagnostic techniques that can be applied to study the conduct of laser induced plasma like shadowgraph, interferometer, selfemission imaging by using fast gated cameras, optical emission spectroscopy (OES), Langmuir probe, Faraday cup, etc. [8].Due to the transient features of the plasma formatted by laser induced plasma (LIP), optical emission spectroscopy (OES) technique with time and space resolution is especially suitable to obtain information about the manner of the formatted species in space and time beside the dynamics of the plasma evolution. Space- and time-resolved OES measurements can be used to calculate the plasma expansion rate, kinetic energy, electron temperature and electron density $[9,10]$. The form and width of the spectral lines emitted by plasma are estimated by collision processes disturbing the released atoms and ions. The principal mechanisms which relating to the broadening of the spectral lines are Stark, resonance, Doppler, and instrumental broadening. The impact of resonance broadening is directly proportional to the ground state number density and the transition oscillator strength. In case of ablation in vacuum, where ablated species show high propagation velocities, one of the predominant contributions to the spectral line broadening is Doppler broadening. This is because of Doppler shifts (i.e., $\Delta \lambda D=\lambda v z / c$ ) due to the different particles in the plume showing various velocity parts $(v)$ in the direction of observation. The effect due to Doppler broadening can be ignored because of its small quantity. Putting the spectrograph at its high resolution can reduce the instrumental broadening. [7]. Stark broadening of spectral lines in plasmas can be attributed to collisions of charged particles, resulting in line broadening and peak wavelength shift. The FWHM of a Stark-broadened line (in angstroms) without an ionic support can be written by the relation

$\Delta \lambda_{1 / 2}=2 \mathrm{~W}\left(\frac{\mathrm{n}_{\mathrm{e}}}{10^{16}}\right)\left(\mathrm{A}^{0}\right)$,

where $\mathrm{W}$ is the electron-impact parameter which can be selected for different temperatures, ne is the electron density $[7,11]$. Electrons released from the target have high kinetic energy and ionize ambient species through collisions. The species excited in such processes as well as participate in various reactions. Thus, electron density and its spatial distribution beside those of the atomic and molecular numbers are

December 2020, Volume 2, Issue 4

p. $42-47$ 
significant variables.Many studies on the electron number density in the plasma plume have been achieved, by using techniques like optical emission spectroscopy, the Langmuir probe, interferometer, and optical transmissivity. The emission spectroscopy has been highly applied due to its noncontact nature of probing and the information accessible over a wide spectral range. The electron density has been calculated from the Stark broadening of emission lines in the case of the laser induced plasma at low pressure conditions [1]. The temporal characteristic of electron density has been achieved to estimate three-body recombination rate constant and recombination time [9]. The purpose of this research is to estimate electron density ne of plasma produced by Nd:YAG pulsed laser of Al element which can be used in many application such as electroptics devices and semiconductors alloys.

\section{Experimental setup:}

The target of the laser induced plasma process is Al with purity $99.999 \%$, and compressed under the pressure (10 tons) to make it shaped liked disc with a diameter of $3 \mathrm{~cm}$ and then sintered in oven to temperature $T$ for 2 hours period. LIP experiment was achieved under vacuum pressure $(0.1 \mathrm{mbar}$ by using Varian DS219 Rotary pump). The beam of Nd: YAG laser with first fundamental harmonic frequency $(\lambda=1064 \mathrm{~nm}, 10 \mathrm{~ns}, 6 \mathrm{~Hz})$ was concentrated onto $\mathrm{Al}$ target with quartz lens $(\mathrm{f}=10 \mathrm{~cm})$, the target was fixed onto rotating motor (speed $4 \mathrm{rev} / \mathrm{min}$ ) to avoid fast crating. The spectroscope used to collect the emission radiation from the plasma was of the type Surwit S3000-UV-NIR. The laser induced plasma experiment was performed at room temperature. LIP setup scheme has been shown in figure (1). Electron density of the AI ablated atoms were computed by analyzing the emission line spectra of the spectroscope data. The Al target was ablated by 1000 pulses. The vacuum pressure was changed from (0.04-0.2) mbar; also the laser pulse energy was selected to be $(800,860,940) \mathrm{mJ}$ to study their influences on the value of electron density.

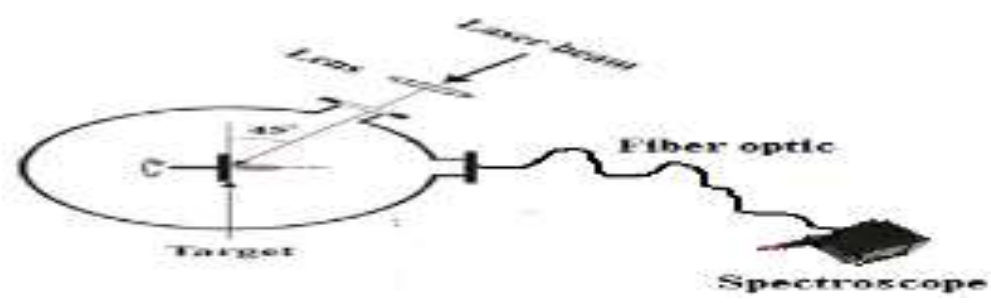

Figure 1. Schematic diagram of the LIP experimental setup.

\section{Results and Discussion:}

In this experiment we took various laser pulse energies (800,860 and 940$) \mathrm{mJ}$ on Al sample, by using the spectroscopy and comparing with NIST database we took a certain peaks of Al (as listed in table 1 ) from the figure between the wavelength and the intensity (figure. 2-4)

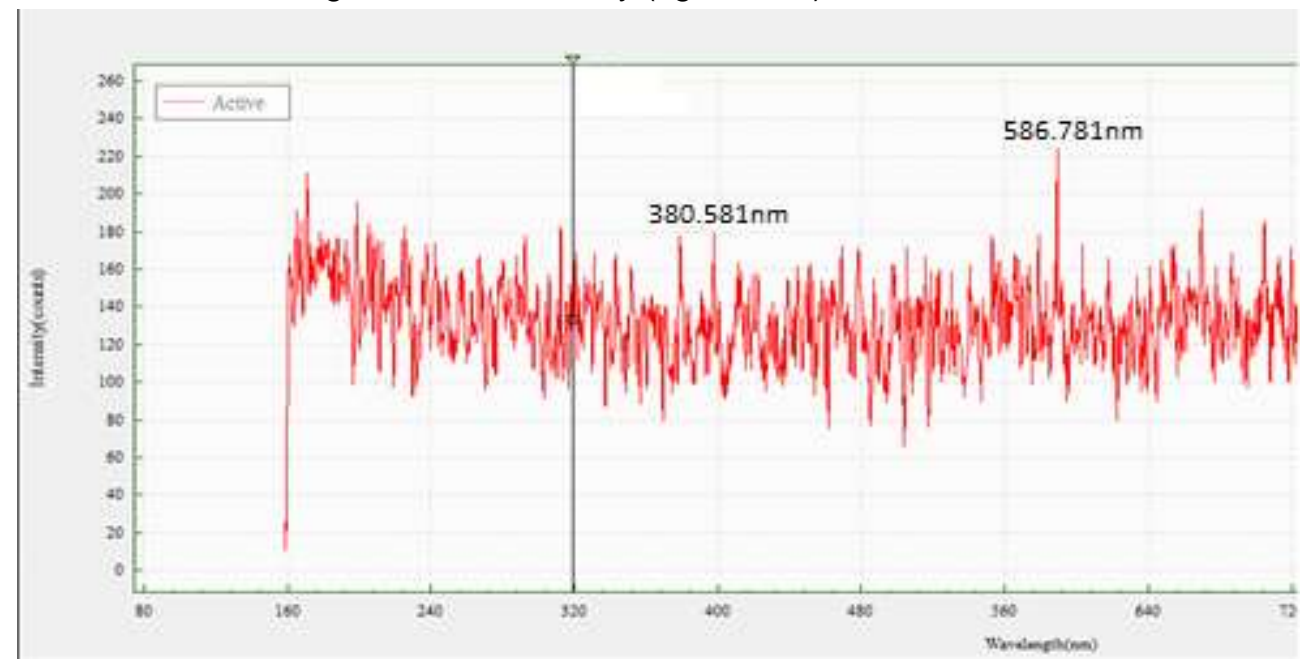

Figure 2. Emission spectrum of Al plasma at laser pulse energy $940 \mathrm{~mJ}$ 


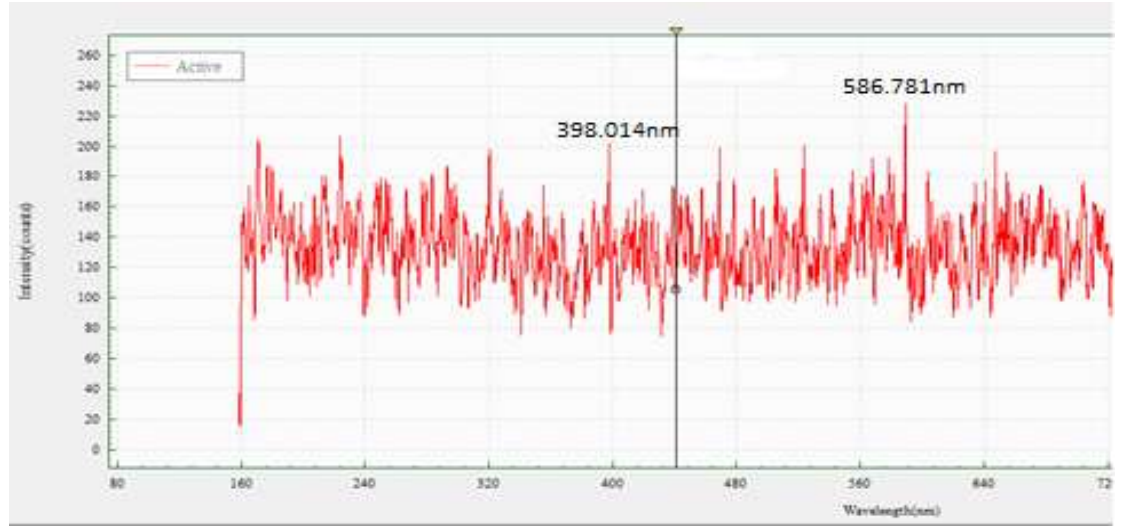

Figure 3. Emission spectrum of Al plasma at laser pulse energy $860 \mathrm{~mJ}$.

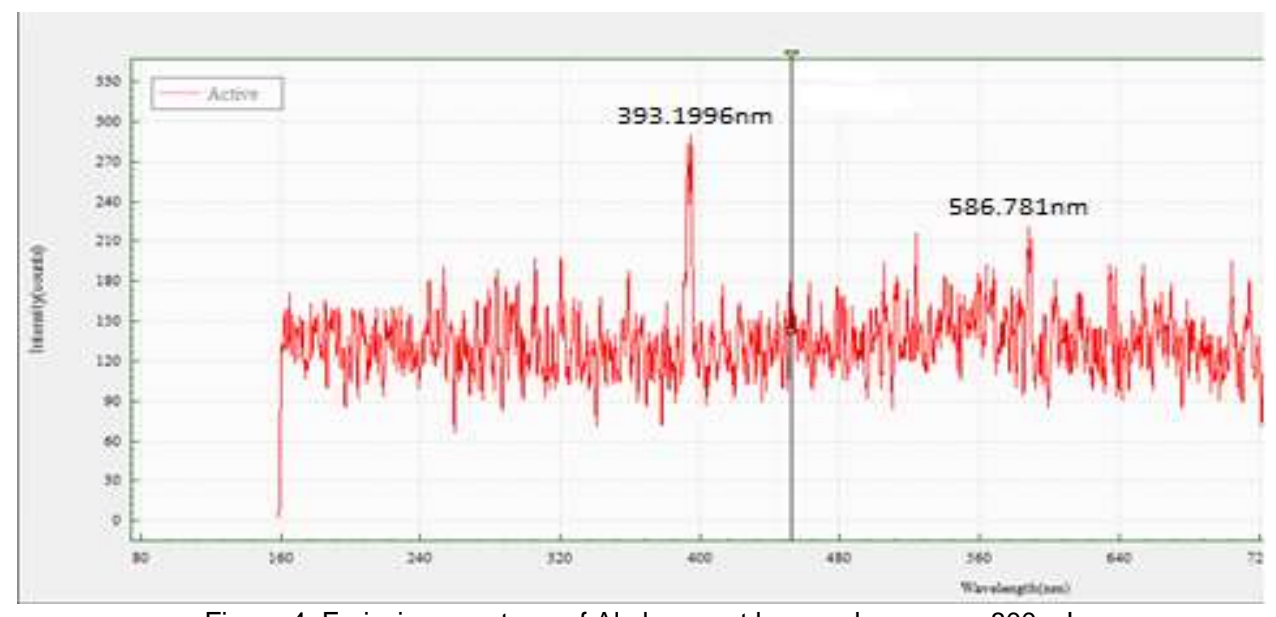

Figure 4. Emission spectrum of Al plasma at laser pulse energy $800 \mathrm{~mJ}$.

We use matlab program and eq.(1) of Stark broadening to calculate the electron density and by taking the full width of half maximum as shown in table 1.

Table 1. Experimental and result data

\begin{tabular}{|c|c|c|c|c|c|c|}
\hline Elaser(mJ) & $\lambda_{1}(\mathrm{~nm})$ & $\lambda_{2}(\mathrm{~nm})$ & $\mathrm{I} 1$ & $\mathrm{I} 2$ & $\Delta \lambda_{1 / 2}$ & $\mathrm{ne}(\mathrm{cm}-3)$ \\
\hline 940 & 380.581 & 586.781 & 178 & 208 & 1.89 & $9.45 \times 1016$ \\
\hline 860 & 398.014 & 586.781 & 203 & 228 & 1.62 & $8.1 \times 1016$ \\
\hline 800 & 393.1996 & 586.781 & 282 & 218 & 0.52 & $2.6 \times 1016$ \\
\hline
\end{tabular}

December 2020, Volume 2, Issue 4

p. $42-47$ 
The proportional relation between the laser pulse energies and the electron has been shown in figure (5).

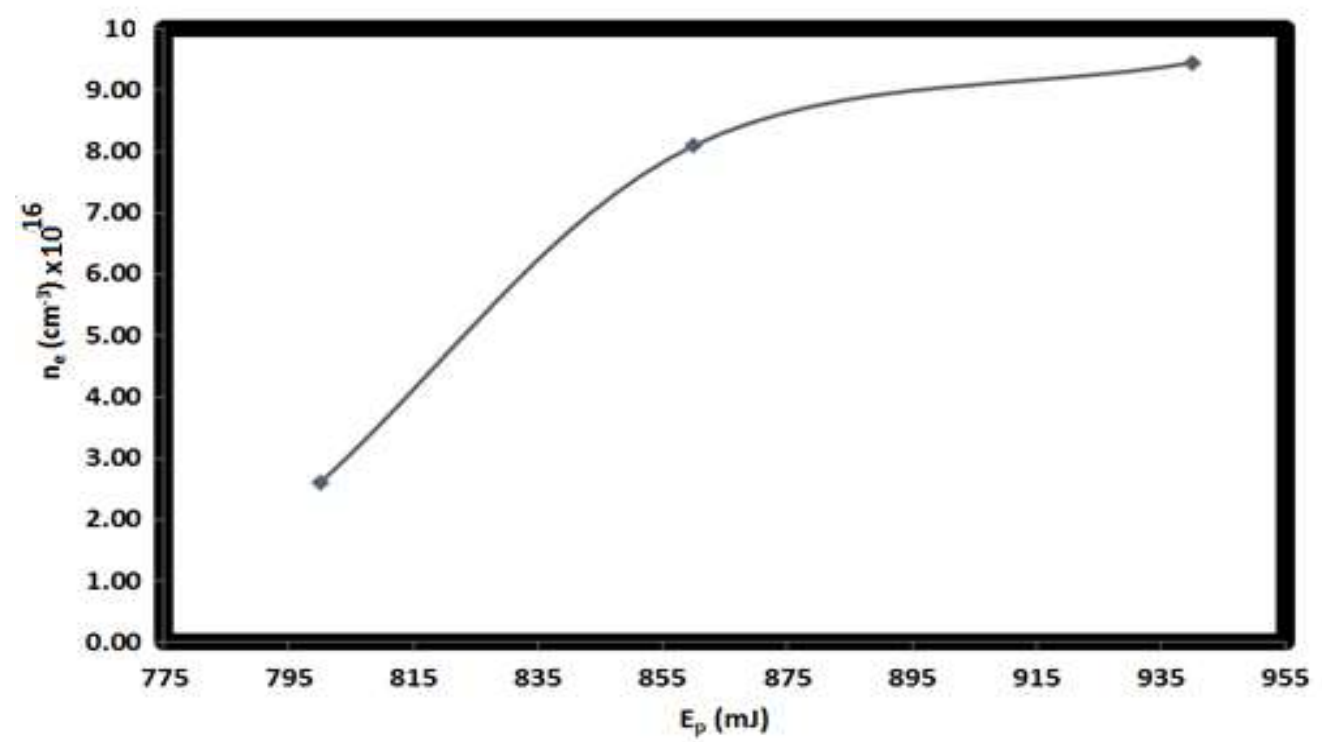

Figure 5. The laser pulse energy versus the electron density.

It is clear from the figure( 5) that as laser pulse energy increases, the electron density increases too. It is proportional relation. The dynamic, fluctuating manner of the LIP is confrontation for diagnostics because the principle plasma quantities change in time and space.

The continuum, or bremsstrahlung, happens when a free electron collides with another particle and makes a transform to another free state of lower energy, with the emission of a photon. In a plasma that is sufficiently hot most of the atom are divested of their orbital electrons, hence making electron-ion recombination and bremsstrahlung the predominant emission mechanisms. The principle mechanisms participating to the broadening of the spectral lines are Stark, resonance, Doppler, and instrumental broadening. The impact of resonance broadening is directly proportional to the ground state number density and the transition oscillator strength. Laser ablation in vacuum, where ablated species show high propagation velocities, one of the predominant contributions, to the spectral line broadening is Doppler broadening. This is because of Doppler shift experience by different species in the plume showing different velocity components toward the direction of observation. Stark broadening of spectral lines in plasma is a result of collisions of charged particles, resulting in broadening of the line and displacement in the peak wavelength. According to such behavior of the electron density ne in figure (5) versus laser pulse energy is attributing to the absorption or reflection of laser energy by the plasma during creation which relies on frequency. By increasing the laser radiation more excited species, ions, and free electrons are obtained and laser pulse interacts with these species, leading to farther heating and ionization. It is observed also from figures (2) to (4) that the width of the lines was increased with the increase of laser energy. In plasma, the spectral lines where naturally broad and the principal sources of the line broadening is Doppler broadening and Stark broadening. It is obvious that the electron density has a maximum value in the starting region of the plasma and decrease toward the direction of expansion. Relatively, the recombination process causes a decrease in the electron number density ne in the widened part of the plasma. It is observed that the electron density ne increases with laser pulse energy, this behavior of the electron density is attributed to the absorption or reflection of the laser energy by plasma and relies on the plasma frequency.

\section{Conclusion:}

A Q-switched Nd-YAG laser with its fundamental harmonic $(1064 \mathrm{~nm})$ has been used to study laser induced Aluminum plasma and measure electron density. The emission spectrum of the plume of plasma discovers transitions of neutral and single ionized atom of Al. the electron density has been calculated toward the axial positions of the plasma plume. The electron density and temperature which close to the target at maximum values. The impact of laser pulse energy on the electron density of laser induced Al plasma has been 


\section{7 | Mohammed R. ABDULAMEER, Ali A-K. HUSSAIN, Kadhim A. AADIM}

verified. As the laser pulse energy increases the electron density increases also, They are directly proportional. Spectroscopic measurement has been used to find electron density by using the technique of line broadening and measure full width of half maximum in order to get the electron density choosing a wide atomic line of Al plasma excited atoms or neutral atoms.

\section{Acknowledgment}

Thanks to the Physics department in University of Baghdad / Plasma laboratory for providing the apparatus and tools for this experiment.

\section{References}

Bhatti, K. A., Khaleeq-ur-Rahman,M., Rafique, M. S., Chaudhary, K. T., and Latif, A. (2010). Electrons emission from laser induced metallic plasmas. Vacuum, 84, 980-985.

Camacho, J. J., Díaz, L., Santos, M., Juan, L. J., and Poyato, J. M. L. (2010). Time-resolved optical emission spectroscopy of laser-produced air plasma. Journal of applied physics, 107, 1-9.

Cvejić, M., Jovićević, S., Mothe, E., Mercadier, L., Konjić, E., and Hermann, J. (2010). Electron density diagnostics of laser induced plasma in helium. Publ. Astron. Obs. Belgrade, 98, 192-198.

Furusawa, H., Sakka, T., and Ogata, Y. H. (2004). Characterization of ablated species in laser-induced plasma plume. Journal of applied physics, 96, 975-982.

Hanif, M., Salik, M., and Baig, M. A. (2012). Diagnostic Study of Nickel Plasma Produced by Fundamental $(1064 \mathrm{~nm})$ and Second Harmonics (532 nm) of an Nd: YAG Laser. Journal of Modern physics, 3, 1663-1669.

Harilal, S. S., O'Shay, B., and Tillack, M. S. (2005). Spectroscopic characterization of laser-induced tin plasma. Journal of applied physics, 98,1-7.

Hussein, A. E., Diwakar, P. K., Harilal, S. S., and Hassanein, A. (2013). The role of laser wavelength on plasma generation and expansion of ablation plumes in air. Journal of applied physics, 113, 1-10.

Khater, M. A. (2013). Influence of laser Pulse energy on VUV emission from laser plasmas under various ambient conditions. Rom. Journ. Phys., 58, 181-192.

Musadiq, M., Amin, N., Jamil, Y., Iqbal, Y., Naeem, M. A., and Shahzad, H. A. (2013). Measurement of electron number density and electron temperature of laser-induced silver plasma. International Journal of Engineering and Technology, 2, 32-43.

Naeem, M. A., Iqbal, M., Amin, N., Musadiq, M., Jamil, Y., and Cecil, F. (2013). Measurement of Electron Density and Temperature of Laser-Induced Copper Plasma. Asian Journal of Chemistry, 25, 21922198.

Tudisco, S., Mascali, D., Gambino, N., Anzalone, A., Gammino, S., Musumeci, F., Scordino, A., and Spitaleri, A. (2011). Investigation of laser-produced aluminum plasma. Nuclear Instruments and Methods in Physics Research A., 653,47-51. 